\title{
Le flou rhétorique dans les textes de brevets américains
}

\section{Monique Frobert-Adamo}

\section{(2) OpenEdition}

1 Journals

\section{Édition électronique}

URL : http://journals.openedition.org/asp/2768

DOI : 10.4000/asp.2768

ISSN : 2108-6354

\section{Éditeur}

Groupe d'étude et de recherche en anglais de spécialité

\section{Édition imprimée}

Date de publication : 1 décembre 1998

Pagination : 99-120

ISSN : 1246-8185

\section{Référence électronique}

Monique Frobert-Adamo, "Le flou rhétorique dans les textes de brevets américains », ASp [En ligne], 19-22 | 1998, mis en ligne le 06 mars 2012, consulté le 30 avril 2019. URL : http:// journals.openedition.org/asp/2768; DOI : 10.4000/asp.2768

Ce document a été généré automatiquement le 30 avril 2019.

Tous droits réservés 


\title{
Le flou rhétorique dans les textes de brevets américains
}

\author{
Monique Frobert-Adamo
}

1 Le présent article est la synthèse de travaux entrepris dans le cadre d'une recherche doctorale. Mes activités d'enseignante angliciste au département chimie de l'IUT A de Lyon, d'une part, et mes interventions dans des entreprises du même secteur en tant que formateur-conseil d'autre part, m'ont conduite à développer une recherche sur la rhétorique des textes de brevets américains dans le domaine des semi-conducteurs.

2 Nous nous intéresserons à l'appareil formel de l'énonciation afin de tenter de cerner le statut intra-textuel des partenaires, dits actants. Puis nous examinerons l'axe rhétorique : certains outils rhétoriques pertinents seront décrits dans le cadre des théories de l'argumentation qui m'ont paru les plus fondamentales. Je m'appuierai sur les 4 extraits du corpus qui figurent en annexe. Ce corpus, qui comprend globalement une trentaine de brevets entre 5 et 120 pages, est organisé de façon diachronique: chaque brevet représente un jalon dans l'évolution des techniques.

\section{Introduction}

3 Le langage des brevets, que nous appellerons dorénavant texte ou texte de brevet, participe uniquement de la production écrite. L'inventeur n'est à aucun moment sollicité pour une communication orale au cours du processus administratif de dépôt et d'obtention. Les normes rédactionnelles législatives sont régulièrement remises à jour et en sont publiées dans le Manual of Patent Examining Procedures. Toutefois, il n'existe aucune contrainte concernant la longueur des descriptions, des comptes-rendus d'expériences ou encore des revendications. Les choix purement linguistiques ne sont pas prédéterminés, ce qui se traduit par une totale flexibilité en matière de développements rhétoriques.

4 Le langage des brevets est un genre. John Swales fait dans Genre Analysis (1990) un certain nombre de remarques qui peuvent s'appliquer au texte de brevet : 
Boyle and his collaborators had to work to make a rhetoric, to develop a convincing style.... It would appear that phenomena only acquire fact-like status by consensus and that consensus may not be achievable without rhetorical persuasion. le texte en tant que langue technique et scientifique. Les paramètres qui en conditionnent l'existence sont: la nouveauté de l'invention, sa reproductibilité et sa protection. Le paradoxe est donc bien là : car, ainsi que le dit Barthes dans Leçon (1978) «La science se caractérise par une rétention du spectacle». Le flou rhétorique en est le résultat perceptible : comment définir et analyser le cadre énonciatif dans lequel il se développe, et justifier l'emploi des outils linguistiques utilisés?

6 La densité du corpus satisfait à deux impératifs, celui de la cohérence diachronique et celui de la longueur. La période englobée court de 1904 à 1994, ce qui permet d'avoir une vision des prémisses de la technologie des semi-conducteurs, de suivre pas à pas les découvertes qui ont conduit à l'apparition des nouveaux matériaux et de procéder à un état des lieux. La langue de spécialité exige du linguiste qui en a fait son secteur d'investigation d'intégrer dans son travail une documentation précise sur les aspects techniques du domaine. Chaque extrait de brevet est précédé d'une fiche signalétique où figurent les caractéristiques administratives, suivies d'une synthèse sur l'enjeu de l'invention ainsi que sur son intérêt stratégique.

7 Comment le linguiste intervient-il dans l'analyse d'un texte de brevet? De fait, son rôle est double: rôle didactique ou comment commenter les paramètres du texte, en particulier lors de séminaires en entreprise, et rôle de veille : le texte de brevet est une source documentaire primordiale dans l'activité de veille technologique.

Dans les deux cas, l'attention se concentre sur les zones du texte où la lisibilité est douteuse, celles précisément où le lecteur doit élaborer des stratégies d'approche qui lui permettront de gérer l'espace de liberté dont le flou rhétorique est à la fois l'agent et le résultat.

Qu'est-ce que le flou rhétorique? Tout d'abord, c'est une construction argumentative, la résultante d'une démarche conditionnée par l'appareil formel de l'énonciation qui fournit le cadre à l'intérieur duquel les outils rhétoriques s'appliquent. Les éléments constitutifs de l'ancienne rhétorique, celle d'Aristote et de la nouvelle rhétorique, celle de l'école américaine avec Perelman et Toulmin, sans occulter les recoupements contestés avec l'approche logique des processus argumentatifs, tous ces ingrédients se retrouvent mêlés dans les textes de brevets : quelques pistes sont proposées dans la deuxième partie de cet article.

10 Si l'on accepte de définir l'argumentation comme «l'opération par laquelle un énonciateur cherche à transformer par des moyens linguistiques le système de croyances et de représentation de son interlocuteur ", le flou rhétorique est inhérent à un discours spécialisé comme celui des brevets.

\section{L'appareil formel de l'énonciation}

11 Voyons quels sont les partenaires dans le transfert des informations contenues dans le texte de brevet. L'inventeur à l'origine de l'existence du texte est le «producteur » du message : il a donc le statut linguistique reconnu de locuteur. Il peut s'agir d'une personne privée, ou d'une équipe qui fonctionne en milieu industriel. 

munauté scientifico-industrielle porte sur le texte une attention à visée technique et stratégique : elle devient dans sa globalité l'allocutaire destinataire du message.

\subsection{Statut linguistique du locuteur}

\subsubsection{Statut linguistique du locuteur : présence explicite}

\section{Degrés de présence dans l'énoncé : affirmation de l'identité}

13 La présence de l'inventeur dans le texte a évolué au cours des décennies. Dans le Brevet $\mathrm{n}$ - 1 (voir Annexes), dont le processus de publication s'est déroulé entre 1906 et 1910, la pronominalisation ne laisse planer aucun doute sur le locuteur :

Be it known that I, Johan Jacob Rink... have invented..., ...having thus described my invention, I claim as new and desire to secure by Letters Patent : ..., ...In testimony that I claim the foregoing as my invention, I have signed my name in presence of two subscribing witnesses.

Les traces visibles de cette présence perdurent dans le Brevet $\mathrm{n}^{\circ} 2$, dont le processus de publication s'est déroulé entre 1937 et 1940 (voir Annexes) :

I therefore particularly point out and distinctly claim as my invention..., I have discovered that..., I do not wish to be limited to the exact procedure set forth...

On remarque que l'enjeu économique et stratégique, au fil du temps, dépasse le cadre strictement individuel pour devenir global. Après une présence du locuteur attestée par une fréquence d'occurrence élevée de la pronominalisation, la tendance à l'effacement se confirme dans des choix linguistiques distanciés. Du locuteur présent dans le texte et clairement identifiable, il y a un glissement vers une présence possible que l'on doit vérifier, et dont la marge d'identification doit être évaluée. Le flou intervient dès qu'il y a incertitude.

Effacement de la présence linguistique du locuteur.

Il suffira de citer des extraits du Brevet $n^{\circ} 3$ (voir Annexes) :

In view of the foregoing it is a principal object of the present invention to provide a method and means for producing inherently curly fibers...

17 La période concernée par le dépôt va de 1957 à 1963. La phrase d'introduction du Brevet $\mathrm{n}$ ${ }^{\circ} 4$ (voir Annexes) obéit aux mêmes impératifs :

The present invention relates to a method for producing a glass preform in the fabrication of an optical fiber...

Ici, la procédure s'est déroulée entre 1992 et 1994.

Toutefois, la présence du locuteur demeure explicite dans le choix des marques indicielles telles que le qualifiant present. L'inventeur est bien au centre du processus qu'il positionne sur l'axe du temps. Il apparaît clairement que la non-présence du locuteur conduit à un enrichissement des outils rhétoriques, ce qui va le conduire à s'exprimer de la manière la plus efficace possible compte tenu de ses objectifs.

\section{Les signaux d'imprévu}


19 La sérendipité, cette faculté de faire, par hasard, des découvertes heureuses et inattendues, annonce un imprévu quelque peu taquin: elle est quasiment neutralisée dans les textes de brevets.

Dans un inventaire des signaux d'imprévu du secteur de la chimie figurent des marques indicielles telles que: unusual, unprecedented, unexpected, anomalous, surprising qui participent de la description de résultats clairement désignés comme inattendus.

21 Le seul exemple de signal d'imprévu dans l'ensemble du corpus se trouve dans le Brevet $\mathrm{n}$ ${ }^{\circ} 4$ : «But, in some cases, bubbles remained in the vitrified preforms, unexpectedly». La technicité et le professionnalisme ont peu à peu gagné du terrain et l'inventeur vit désormais sous contrat de réussite. La législation lui a retiré un trait essentiel : l'imprévu, un des ingrédients de la découverte. Le flou rhétorique en est-il la reconstruction?

\subsubsection{Statut linguistique du locuteur : présence indirecte ou implicite}

\section{Expressions interprétatives, évaluatives, modalisatrices (hedges)}

Selon Swales (1990) ces expressions sont associées à «cautiousness, rhetorical diffuseness »; ce sont des « rhetorical devices for projecting honesty, modesty, and for diplomatically creating space ».

Voyons quelques exemples. Dans le Brevet $n^{\circ} 2$ :

It more particularly concerns a method of purifying chlorine containing relatively small amounts of organic impurities..., ...the chlorine still contains relatively small amounts, generally less than about 1 percent by weight, of organic impurities...

Les hedges sont autant de ressources du lexique qui agissent comme des curseurs dans le cadre de l'énonciation. Comment contraster «small » et « relatively small »? Au-delà du phénomène de récurrence, la description de l'invention progresse, le locuteur fournit des indices, puis le texte est verrouillé par " generally less than about 1 percent by weight. »

Lorsque l'inventeur déclare :

Be it known that I, Johan Jacob Rink, have invented certain new and useful improvements... (Brevet $\left.\mathrm{n}^{\circ} 1\right)$,

ou encore :

In the application referred to, there is described an apparatus in which the film of mercury which forms the cathode lies upon the bottom of a suitable vessel, so that amalgamation takes place only at the upper surface of such film ...

nous constatons que ces expressions sont nécessaires pour structurer l'espace d'interprétation, ce qui, par effet retour, assure pour le locuteur une forme de protection : la flexibilité que confère au texte l'introduction des hedges permet à ce texte de plier sans rompre.

Outils rhétoriques: expression de la prudence, projection de l'authenticité et création d'un espace de liberté : enthymèmes et syllogismes

L'enthymème - du grec du grec enthumêma « ce qu'on a dans l'esprit » - est devenu terme de rhétorique et désigne un syllogisme fondé sur le probable. Le mot a conservé le sens dans 
son étymon et désigne, en logique, une forme abrégée de syllogisme dans laquelle on sous-entend l'un des deux prémisses, ou la conclusion.

Le syllogisme est un terme qui nous vient de la logique aristotélicienne : il désigne une suite de trois propositions telles que l'accord des deux premières (prémisses) exclut le refus de la troisième (conclusion). Chaque proposition est constituée par un sujet, un prédicat et une copule qui les lie : la conclusion est possible parce qu'un terme commun aux deux prémisses permet de relier les deux autres termes.

On a donc le modèle : $\mathrm{X}, \operatorname{donc} \mathrm{Y} . \mathrm{Z}$ doit être vraie.

Voici concrètement comment ces outils interviennent dans le décodage du texte :

The invention, then, consists of the method hereinafter fully described and particularly pointed out in the claim, the annex drawing and the following description setting forth a mode of carrying out the invention... $=\mathrm{X}$

...such mode illustrating however but one of the various ways in which the principle of the invention may be used. » = donc $\mathrm{Y}$.

Fuzziness/ Flou : ...but one of the various ways......may be used.

$\mathrm{Z}=$ there are other ways to use the principle of the invention: le flou rhétorique persiste dans la construction même du raisonnement.

Selon Oswald Ducrot (1991), une des conditions d'existence de l'énonciation est que l'implicite soit perçu comme un prolongement du niveau explicite. La signification implicite est reconstituée par raisonnement par l'allocutaire, alors que la signification littérale est la responsabilité du locuteur. Le syllogisme est un procédé qui s'appuie sur l'organisation interne de l'énoncé : il laisse inexprimée une affirmation nécessaire à la cohésion de cet énoncé.

\subsection{Statut linguistique de l'allocutaire}

\subsubsection{Code de présentation du texte}

\section{Relation de compétence entre les partenaires : flou par défaut ?}

Le texte de brevet est doté d'une structure type dont la codification s'est affinée au cours des décennies. Les textes du début du siècle étaient rédigés en continu; le brevet américain comporte aujourd'hui un abstract de présentation, accompagné de toutes les références d'antériorité; on trouve ensuite les noms de l'inventeur et/ou de l'entreprise ainsi que la date dépôt suivie de la date d'obtention (voir fac-similé en Annexe). Le brevet lui-même débute par une « Description of the Related Art » qui cautionne la relation de compétence entre les partenaires :

Methods for forming continuous and discontinuous curly glass fibers have been developed in the past, but in each such known methods, some time after treatment is required...

Glass fibers are known to have weakness to abrasion...

31 Mais, seule une connaissance du contenu des schémas allusifs peut permettre à l'allocutaire d'accéder au sens. De plus, l'évaluation que le locuteur peut faire de cet accès au sens est fondée sur des présupposés. Le texte s'adosse au déjà-connu et trouve sa justification dans l'état de l'art. Le niveau d'acceptabilité sera d'autant plus élevé que le champ défini est large et que les points d'appui sont multiples.

Renvoi à la fonction phatique selon Jakobson : flou par excès? 


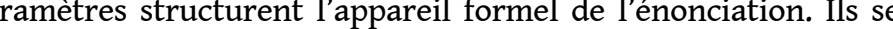
maintenir la communication, donc finalement peu importe leur caractère d'incomplétude. On sait depuis Jakobson que cette accentuation du contact - la fonction phatique - donne lieu à un échange (?) profus de formules ritualisées.

A feature of the invention lies in the fact that...

A further feature of the invention lies in the adaptability of the invention... A still further feature of the invention lies in the lack of need for...

Another and still further feature of the invention lies in the fact that... (Brevet $\mathrm{n}^{\circ} 3$ )

Et pourtant, le locuteur cautionne le flou par excès quand il déclare : "These and other objects and features of the invention will become more apparent... ». Il y a donc un manque à combler. Ainsi que le mentionne Catherine Kerbrat-Orecchioni :

Il arrive, ainsi que le remarque Genette, que, dans certains types de textes, la figure fantomatique de celui qu'il appelle le «narrateur » détermine l'attitude du locuteur, vienne en quelque sorte hanter, habiter tous les replis du texte. (1980:159)

\section{Présupposé de l'intégration à une communauté}

Toute construction rhétorique a une visée persuasive, ses points d'ancrage doivent par conséquent être clairement identifiés. La construction triadique locuteur-allocutairetexte présuppose que le locuteur et l'allocutaire sont intégrés à une communauté et que cette intégration génère les canaux de communication. D'où l'existence d'un code et l'identification des composantes du cadre du schéma énonciatif. Sans oublier la situation où les partenaires se confondent: nous ne sommes pas très éloignés de «l'énonceur psychosocial » au sens où l'entend Claude Hagège dans L'Homme de Paroles :

L'énonceur psychosocial réduit en lui-même tous les types d'usages de la langue en fonction des situations. C'est pourquoi les distinctions d'inspiration logicosémantique ne sont pas toujours opérantes si l'on entend le saisir dans sa réalité, c'est-à-dire dans une perspective discursive et textuelle. (1985:31)

\subsubsection{Métalangage et flou rhétorique}

\section{Repérages des traces dans l'énoncé}

En s'adossant au déjà-connu, le locuteur fait référence à un métalangage qui englobe le flou (fuzziness) inhérent à la construction de la réalité.

Les traces de l'inscription dans l'énoncé s'estompent :

In the electrolytic production of chlorin in the usual types of electrolytic cells and after the chlorin delivered by the cells has been subjected to conventional preparation.... (Brevet $\left.\mathrm{n}^{\circ} 2\right)$

In a conventional method for producing a glass preform for an optical fiber, a glass soot preform is produced by a vapor phase synthesis method such as the VAD method or an OVD method.... » (Brevet $\left.\mathrm{n}^{\circ} 4\right)$

37 C'est donc à partir de ces traces dans l'énoncé que l'allocutaire va reconstruire un référentiel qui est le métalangage du texte de brevet : cette reconstruction est vitale pour le locuteur-inventeur, car le métalangage est le topos de communication. Voyons les précisions apportées par Christian Plantin :

On trouve le premier inventaire des lieux argumentatifs dans les Topiques d'Aristote, ainsi que leur définition, qui les met dans la dépendance de la théorie de la prédication. À la suite d'Aristote, la rhétorique ancienne va postuler une 
structure universelle commune à tous les événements possibles et, homologiquement, à toutes les disputes imaginables pouvant survenir à leur sujet. La théorie des lieux ou «topiques " répertorie et circonscrit le pensable, la forme générale des jugements, donc des énoncés formulables en une occasion quelconque. $(1990: 236)$

\section{Métalangage et communication}

Au-delà de la phrase se développent les figures de style dont je ne chercherai pas à faire ici un inventaire exhaustif. Chacune d'elles contribue à transférer du sens en partant du niveau concret, celui de la description et de l'expérimentation, pour aller vers le niveau abstrait, celui où interviennent les schémas mentaux. Il est important de ne pas amalgamer le flou, le vague et l'ambiguïté. Le flou est en étroite relation avec la manière dont nous construisons nos systèmes conceptuels. C'est le flou omniprésent dans le langage qui nous permet d'élucider les aspects signifiants de la communication.

We communicate not to exchange accurate information, nor to look for a single comprehension of meaning, but to interact using the largest possible variety of fuzzy linguistic facets co-existing in parallel and complementing one another. (Dimitrov \& Russell)

Citons pour mémoire :

L'hyperhypotaxe, qui consiste à insérer des subordonnées en trop grand nombre et, ce faisant, un nombre excessif d'idées connexes. L'hyperhypotaxe est une période destinée à être lue. Son développement épouse la diversité et la multiplicité des rapports perceptibles lors d'une lecture, alors que la période classique correspond au rythme soutenu oral :

$$
\begin{aligned}
& \text { Optionally, the soot preform which has been heated in the first heating step } \\
& \text { under reduced temperature at a temperature higher than the temperature in } \\
& \text { the first heating step and lower than the vitrification temperature, before } \\
& \text { the vitrification of the preform. (Brevet } \left.n^{\circ} 4\right)
\end{aligned}
$$

Dans les cas extrêmes d'hypotaxe "mal bâtie ", on a une synchise: pour la rhétorique classique, la synchise est un défaut qui consiste à rompre le déroulement syntaxique par des sortes de parenthèses qui laissent en suspens la construction. Je reviendrai plus loin sur la question des imperfections du texte. L'étoffement de la structure syntaxique vise la complexification.

Le brouillage syntaxique me semble fort bien nommé.(voir 1.2.4.)

Le bouleversement syntaxique rend, quant à lui, la phrase inintelligible.

\subsubsection{Structure des schémas d'inférence}

\section{Stratégies d'interprétation : logique flou et complexité}

43 Jayez (1988) a mis en évidence la nécessité d'intégrer le problème de la compréhension textuelle dans une analyse sur les schémas d'inférence. Les systèmes de représentation sont, par nature, risqués et imparfaits. Les connecteurs, par exemple, fonctionnent comme des « filtres à inférence ». Le cas de and illustre un filtrage complexe qui nécessite une étude en soi :

In the production of the glass preform, it is essential to reduce or completely remove the bubbles from the glass preform and make the outer diameter uniform. (Brevet $\left.n^{\circ} 4\right)$. 

Don conative trouve son expresion grammaticale la plus pure dans le vocatif et l'impératif. S'il est vrai que dans ses Essais de Linguistique Générale, Jakobson concentre son propos sur la communication verbale, il n'en demeure pas moins que le texte de brevet porte les traces de la fonction conative :

It will be understood that variations of the concepts herein presented will be readily discernible by those familiar with the glass fiber art, and (it will be understood) that modifications can be made in the structures and processes within the broad concepts of the invention.... (Brevet $n^{\circ} 3$ )

From the foregoing description of one mode carrying out the invention, it will be readily appreciated that various modifications are possible and I do not wish to be limited to the exact procedure set forth. (Brevet $n^{\circ} 2$ )

\subsubsection{Les imperfections du texte}

Elgificantive au flou rhetorique, toute la question etant de savoir si ces inperfections sont significative au flou rhétorique, toute la question étant de savoir si ces imperfections sont effectivement « construites » au sens où la rhétorique est une construction du discours, ou bien si elles sont les manifestations, disons, d'un certain type d'ignorance ou d'un manque de maîtrise de l'outil linguistique.

\section{Figures rhétoriques, redondances et complexité}

L'hypotaxe: on rapproche un grand nombre de groupes syntaxiques en indiquant leurs liens de subordination, mais aussi de coordination, ce qui conduit à des empilements et des redondances :

Correspondingly, films or flakes of two glasses might be formed wherein one side of the form is of one glass, and the other side is another glass to impart a curly or wavy character to the form.

Electrical terminals are provided at opposite ends of the length of the feeder in conventional manner and electrical current is passed therethrough to heat the molten masses contained in the feeder, but the lower portion of the feeder is baffled, or in a sense bridged widthwise so that each glass extending through the lengthwise half has access to the full width of the feeder in alternate zones along the length of the feeder. (Brevet $n^{\circ} 3$ ) 
La brachylogie : tous les Noun Phrases en sont l'illustration, dans la mesure où ils sont « une manière abrégée de s'exprimer ». La fréquence d'occurrence de ces structures augmente au fil du temps ; il me semble toutefois qu'une approche statistique, à laquelle je ne me suis du reste livrée que de façon très intuitive et par conséquent fragmentaire, ne peut contribuer de façon convaincante à une étude du flou rhétorique dans les textes de brevets. En effet, la densité de telle ou telle manifestation du discours ne peut constituer une preuve en soi irréfutable du caractère délibéré de son utilisation. En revanche, son positionnement à un moment donné de l'énonciation me semble intéressant en termes de stratégie rhétorique.

On trouvera ci-après des exemples choisis dans le Brevet $n^{\circ} 1$ :

«the electrolyte receptacle» $\rightarrow$ deux reformulations sont envisageables: «the receptacle that contains the electrolyte» ou «the receptacle that is intended for the electrolyte».

«the chlorin gas» -> deux reformulations sont envisageables: «the gas which is designated as chlorin » ou " the gas which contains chlorin ».

\section{Utilisation de l'espace de liberté : degré d'informativité ?}

On voit donc que la complexité générée par les imperfections du texte rejoint la question des stratégies d'interprétation. Le langage introduit des déformations: c'est un trait constitutif. Le sens d'une phrase constitue un ensemble d'indications que ses éléments fournissent pour construire des contextes dans lesquels elle sera interprétable. C'est un trajet vers la conclusion.

Une question se pose : à partir des indications qui gèrent le rapport argumentatif entre énoncés, peut-on émettre l'hypothèse qu'il existe des méta-règles d'inférence et faire ainsi un premier pas vers un modèle de représentation du flou rhétorique?

\section{L'axe rhétorique}

À ce stade de mon travail, le modèle est en cours d'élaboration. Plusieurs pistes semblent converger. Je vais en présenter succinctement trois.

Roger Bautier déclare fort justement :

L'argumentation est en effet à la fois raisonnement (établissement de liaisons entre propositions) et influence (exercice de pressions ou de sollicitations pour faire accepter une position); elle allie le souci de la rigueur avec la nécessité de traiter des concepts flous; elle suppose qu'un accord est possible mais doit tenir compte des divergences irr_ductibles entre personnes et entre groupes; elle est censée respecter la vérité et contribuer à l'établir, tout en recherchant l'efficacité, qui implique l'utilisation de toutes les armes. (1994: 83)

\subsection{Typologèmes et persuasion}

54 Tout genre est porteur de typologèmes que l'on peut définir comme les axes distinctifs hétérogènes de la rhétorique. Dans le cas des textes de brevets, l'axe rhétorique est à effet persuasif :

- persuasion de la nouveauté (novelty)

- persuasion de la faisabilité, reconnaissance du statut de l'inventeur et de son invention ( acceptance or rejection) 


\section{l'inventeur à lancer un double appel :}

- appel à l'usage (appeal to common practice)

- appel à l'autorité (appeal to scientific experts)

Voyons quelques illustrations concrètes des analyses les plus représentatives de la théorie de l'argumentation.

\subsubsection{Exemple d'argumentation : Brevet $n^{\circ} 3$}

\section{Le modèle de Toulmin : présentation et discussion}

57 Ainsi que l'on peut le vérifier en Annexe, ce texte met en scène la mise au point d'une méthode destinée à améliorer les performances des fibres de verre : l'intérêt stratégique en 1963 est de taille.

The Uses of Argument de Stephen Edelson Toulmin a été publié en 1958. L'ouvrage est

marqué par les postulats méthodologiques de l'analyse linguistique qui caractérisait la philosophie anglaise des années quarante et cinquante. (Plantin $1990: 26)$

59 Toulmin constate la pluralité des domaines de l'argumentation, diversité clairement perceptible dans la marque de pluriel du terme uses : je voudrais donc concentrer mon propos sur le chapitre central de son ouvrage "The layout of argument », où est proposé un schéma détaillé de la démarche argumentative. Toulmin critique fortement la validité formelle qui ne garantissait ni la valeur ni l'intérêt d'un raisonnement; ce qui nous ramène à l'espace de liberté du cadre énonciatif et l'adhésion à cet espace que le locuteur cherche à susciter chez l'allocutaire. L'assertion rationnelle «entre dans un réseau complexe d'énoncés » (Plantin 1990 : 26). Le modèle présente les différentes étapes d'un schéma procédural (layout).

\section{Argumentation basée sur la cause}

Un discours argumentatif vise à intervenir sur les opinions, attitudes ou comportements d'un interlocuteur ou d'un auditoire en rendant crédible ou acceptable un énoncé ( Conclusion) appuyé, selon des modalités diverses, sur un autre, appelé argument (Data). Le schéma de base selon Toulmin est une mise en relation des données avec une conclusion. Un schéma d'inférence simple permet d'en tirer une loi, dont l'acceptation par l'allocutaire constitue le but ultime du locuteur.

61 Entre les Données (Data) et la Conclusion (Conclusion) du raisonnement, la Loi de passage ( Law) complète le schéma argumentatif.

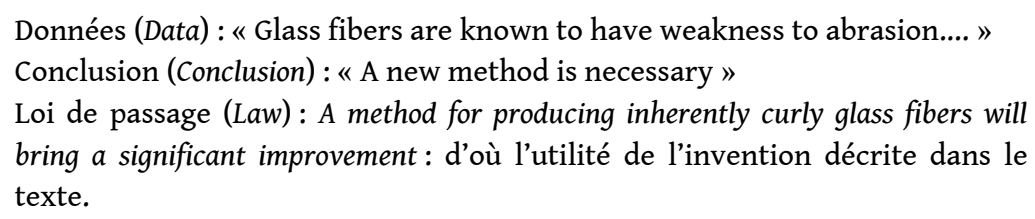

Toutefois, cette Loi étant du domaine de l'inférence, le locuteur ne contrôle pas totalement son passage : Toulmin fournit une règle, une 'licence d'inférer', dite 'warrant' (L). «Les données factuelles prennent statut d'argument». (Plantin op. cit: 27). Mais, étant donné que (L) et (D) ne permettent évidemment pas d'inférer avec un degré absolu de certitude, Toulmin introduit des indicateurs de force $(\mathrm{F})$ et de restriction (R) : ce modèle peut donc être contesté, ce qui est, somme toute, rassurant. 
63 L'introduction du doute dans le champ argumentatif nous amène à nous interroger sur le degré de vérité d'une assertion : cette vérité ne serait plus binaire, mais graduelle. Que l'on se situe dans la perspective d'une approche vériconditionnelle ou que l'on privilégie, ainsi que Toulmin l'a fait ultérieurement, les aspects interactifs de l'argumentation, il semble bien que le flou rhétorique puisse réconcilier ces deux types d'analyse.

D : Glass fibers are known to have weakness to abrasion,

C : (therefore) a new method is necessary,

$\mathrm{F}$ : unless no significant improvement is required

$\mathrm{R}$ : unless no money is available/ unless no applications are found

Un Support (Backing) peut être nécessaire pour la Loi :

A new method for producing ...will bring significant improvement since no significant improvement has been observed yet, as we can see in previous patents/as we can see in the background of the invention.

Cet effet multifacettes d'une réalité sans cesse construite et déconstruite participe de l'élaboration du flou rhétorique.

Argumentation basée sur une classification

De la même manière, nous avons :

Données (Data) : « Glass fibers have limited extensibility. » (D)

Conclusion: «This is a limitation preventing their adaptability for specific applications. » (C)

Loi de passage (Law) : Glass fibers lack adaptability for specific applications : d'où

le caractère de nouveauté du dépôt de brevet. (L)

Le schéma argumentatif, nous l'avons vu, contient une régulation potentielle de sa conclusion. L'énoncé D ne prend son statut d'argument pour C qu'en fonction de L. C'est en fait sur L que repose l'édifice argumentatif. (voir Plantin 1990 : 29)

67 Je conclurai cette approche du modèle de Toulmin en faisant remarquer qu'on y trouve une analyse tout à fait intéressante des argumentations secondaires qui se greffent sur les argumentations principales.

\subsubsection{Réseaux déictiques et anaphoriques : Brevet $n^{\circ} 2$}

Dans l'extrait du Brevet $n^{\circ} 2$ que nous envisageons ici, l'information est présentée sous forme d'un réseau dense de retour sur les données. Les concepts centraux pour la validation de l'invention sont repris de façon récurrente.

I therefore particularly point out and distinctly claim as my invention :

The process of removing the organic impurities from impure chlorine gas containing less than about 1 per cent by weight of such impurities, which comprises introducing the impure chlorine gas into the base of a scrubbing tower below the level of the impure chlorine gas therein collected at a later step, permitting the chlorine gas to bubble through the said impure liquid chlorine and ascend in the tower against a downward flow of a relatively small amount of pure liquid chlorine produced in a later step and introduced into the upper portion of the said scrubbing tower whereby the organic impurities are absorbed by the liquid chlorine forming an impure liquid chlorine which collects in the base of the said scrubbing tower for use in the first step, withdrawing the so-treated chlorine gas from the upper portion of the scrubbing tower and impure liquid chlorine from the base thereof, 
liquefying the chlorine gas so withdrawn, and supplying the upper portion of the scrubbing tower with liquid chlorine from that so liquefied.

69 Cette stratégie rhétorique a un double rôle: elle assure la cohésion de la séquence informative et elle guide le lecteur dans sa compréhension et son interprétation.

\section{L'approche systémique fonctionnelle de MAK Halliday}

Dans son Introduction to Functional Grammar, MAK Halliday (1994) ne se positionne pas comme un spécialiste de l'argumentation. Ses travaux ont néanmoins largement contribué à faire progresser la réflexion linguistique dans ce domaine: il prend en compte aussi bien les paramètres logiques d'organisation de la phrase que les interactions entre les idées, les faits et la syntaxe. Il explore les relations extratextuelles et les projections de processus mentaux sur la reconstruction du réel.

\section{Le concept de cohésion}

71 Voici quelques extraits de Halliday (1994: 309) :

It is necessary also to make explicit the external relationship between one clause and another, and to be able to do so in a way which is not dependent on grammatical structure...

But the clause complex has certain inbuilt limitations...

But in order to construct discourse we need to be able to establish additional relations within the text that are not subject to these limitations...

These non-structural resources for discourse are what are referred to by the term Cohesion.....

72 Le flou est précisément ce qui échappe au cadre et aux limites de la phrase. La reconstruction des éléments et des chaînons manquants conduit-elle à cette cohésion? Les réseaux déictiques et anaphoriques en sont-ils la trace visible dans l'énoncé ? Le modèle de Toulmin reflète les mêmes préoccupations que l'expansion chez Halliday dans son chapitre Above the Clause. Il y aborde, entre autres, les métaphores conceptuelles : elles contribuent à la cohésion. C'est en construisant la variante congruente d'une phrase donnée que l'on voit sa densité lexicale diminuer, et donc la cohésion textuelle. Ce qui tendrait à démontrer que les formulations non congruentes, ou métaphores conceptuelles, qui recèlent les zones de flou, contribuent à la cohésion du texte.

\subsection{Les limites : la complexité et l'approche logique}

73 À ce stade del'analyse, les différentes caractéristiques du texte de brevet convergent pour rendre compte de son fonctionnement. Ceci suppose un idéal logique du discours de la science. Qu'en est-il?

74 Cette complexité aux multiples facettes est-elle une limite à l'efficacité rhétorique ? de même, l'approche logique paraît infiniment réductrice, on le sait, à la plupart des rhétoriciens. Les remarques suivantes visent à apporter un éclairage croisé sur ces questions.

\section{Les tautologies}

Les grammairiens appellent tautologie cette répétition des mêmes choses, qui ne sert qu'à rendre le discours plus long et, dans certains cas, plus ennuyeux. Lorsqu'on dit beaucoup plus qu'il n'est nécessaire et que le discours est chargé de paroles superflues, ce défaut est alors nommé périssologie. Nous rejoignons ici le flou par excès. 


\section{En effet :}

- Il s'agit d'influencer l'auditoire : cette notion tout à fait fondamentale différencie les approches logiques structurales et les approches rhétoriques de l'argumentation. Le rédacteur du texte qui a le statut d'inventeur, ou qui assiste ce dernier, met en oeuvre des techniques discursives « permettant de provoquer ou d'accroître l'adhésion des esprits aux thèses qu'on présente à leur assentiment. »

- La visée argumentative se définissant en termes d'influence plus ou moins forte exercée sur l'auditoire, le but de l'argumentation n'est pas d'approcher au plus près une vérité préétablie. L'auditoire est donc donateur de vérité : il est destinataire et évaluateur des actions rhétoriques. La notion même d'auditoire, qui se trouve à la racine de la rhétorique du texte, est floue.

- Le texte de brevet s'adresse à des auditoires restreints de spécialistes. Comment rendre compatible la rationalité d'un domaine spécifique de compétence avec la notion d'auditoire universel ? Le travail ciblé sur chaque unité-texte devient alors le seul possible.

Perelman conçoit le mouvement rhétorique comme un transfert d'adhésion. L'argumentation ne transfère pas des prémisses vers une conclusion une propriété objective, telle que la vérité, ce qui est le cas de la démonstration, mais s'efforce de faire 
passer vers la conclusion l'adhésion accordée aux prémisses. Ce qui nous satisfait, car il y a alors place pour l'élaboration du flou.

Tout ceci présuppose un idéal logique du discours dont le texte de brevet n'est pas un parangon.

\section{La validité des choix rhétoriques}

L'étude des choix rhétoriques ne peut se satisfaire d'aborder uniquement le message et son contenu ; il faut se préoccuper de la manière dont l'information est transférée et des aspects sociologiques de la communication.

que la communication est dépendante de l'intention, du contexte et de la convention nous conduit à rejoindre Halliday et ses analyses en profondeur des mécanismes de la langue.

5 Les carences du texte de brevet sont de deux natures: segments volontairement agrammaticaux, segments aberrants par négligence, nous l'avons vu en (1.2.4). Ces éléments feront l'objet d'une autre étude; toutefois, ils suscitent de multiples interrogations pour le lecteur et contribuent à renforcer l'impression de flou.

Les choix rhétoriques dont l'analyse est proposée dans cet article rejoignent en majorité les canons de la rhétorique aristotélicienne : invention, arrangement, style, expression, mémoire. Une question se pose désormais: l'approche diachronique permettant de constater la permanence des choix validés par l'histoire, ces choix sont-ils preuves que le texte est articulé sur une rhétorique de la protection dont le flou participe?

Les exemples sont essentiels dans les dépôts de brevets. Leur accumulation est une façon supplémentaire d'assurer la protection de l'invention. Ils sont aussi moyens de réfutation. Ils entrent dans l'histoire sans cesse reconstruite de la technique.

Le flou est ce qui permet cette reconstruction et l'évolution qui l'accompagne. Le flou fait évoluer le texte selon un double mouvement: vers le passé et vers l'avenir. Le flou est l'accélérateur et le frein du texte.

Définition du flou, telle qu'elle est donnée par Le Dictionnaire Historique de la Langue Française :

Nom repris (1976) de l'ancien français flo/ flou qui signifiait inculte, désert (1200). Mais flou est d'abord repris comme terme d'art: Félibien écrit (1676) : «C'est un vieux mot dont autrefois on se servait pour exprimer en termes de peinture, la douceur et la tendresse d'un ouvrage. Par extension, il prend le sens d'indécis, indistinct, » sens où il s'est substantivé (le flou, 1904). Flou qualifie et désigne spécialement $\left(\mathrm{XX}^{\mathrm{e}} \mathrm{s}\right.$.) en photographie, au cinéma, l'effet obtenu volontairement en diminuant la netteté des images.

L'inventeur est un stratège-poète.

\section{La sémiosis illimitée selon Umberto Eco}

90 Umberto Eco remarque, dans Les limites de l'interprétation (1990):

Quand Peirce propose sa définition du lithium sous forme d'un paquet d'instructions destinées à rendre possible non seulement l'identification, mais aussi la production de lithium, il faut remarquer que « The peculiarity of this definition is that it tells you what the word lithium denotes by prescribing what you are to do in order to gain a perceptive acquaintance with the object of the world. »

91 Le seul problème est que l'attitude de l'allocutaire, comme nous l'avons vu, ne s'arrête pas là. La lecture du texte de brevet ressemble fort à l'identification d'un phénomène sémiosique tel qu'il est décrit par Eco dans La structure absente (1972) : 
On a un phénomène sémiosique lorsque, à l'intérieur d'un contexte culturel donné, un objet donné peut être représenté par le terme 'rose' et le terme 'rose' peut être interprété par 'fleur rouge', ou par l'image d'une rose, ou par une histoire entière qui raconte comment on cultive les roses.

L'invention, les façons de la raconter et les stratégies déployées pour la protéger, obéissent à cette acception. Chaque degré dans l'interprétation est franchi, rendu accessible et transgressable par le chemin du flou.

\section{Conclusion}

L'aboutissement au degré zéro de l'écriture conduit à émettre l'hypothèse que le texte de brevet s'apparente aux pratiques incantatoires de la magie qui, du reste, possède un arsenal d'outils rhétoriques parfaitement identifiés : le texte de brevet est un langage initiatique.

Le flou serait-il une composante du secret des alchimistes?

\section{BIBLIOGRAPHIE}

Barthes, R. 1978. Le degré zéro de l'écriture. Paris : Seuil.

Barthes, R. 1978. Leçon. Paris : Seuil.

Bautier, R. 1994. De la rhétorique à la communication. Grenoble : Presses Universitaires de Grenoble.

Ducrot, O. 1991. Dire et ne pas dire: Principes de sémantique linguistique. Paris : Hermann.

Eco, U. 1972. La structure absente: Introduction à la recherche sémiotique, Paris : Mercure de France.

Eco, U. 1990. Les limites de l'interprétation. Paris : Grasset.

Hagège, C. 1985. L'homme de paroles : contribution aux sciences humaines. Paris : Fayard.

Halliday, M.A.K. 1994. Introduction to Functional Grammar. London : E. Arnold.

Halliday, M.A.K. \& J.R. Martin. 1988. « On the language of physical science ». In Registers of Written English, Chapter 3. Londres : Pinter, 55-68.

Jayez, J. 1988. L'inférence en langue naturelle. Paris : Hermès.

Kerbrat-Orecchioni, C. 1980. L'énonciation : De la subjectivité dans le langage. Paris : Armand Colin.

Perelman, Ch. \& L. Olbrechts-Tyteca. 1958. Traité de l'argumentation, la nouvelle rhétorique, Paris : Presses Universitaires de France.

Plantin, Ch. 1990. Essais sur l'Argumentation: Introduction à l'étude linguistique de la parole argumentative. Paris : Éditions Kimé.

Robrieux, J-J. 1993. Éléments de Rhétorique et d'Argumentation, Paris : Dunod.

Salem, J. 1987. Introduction à la logique formelle et symbolique. Paris : Éditions Nathan. 
Swales, John. 1988. « Discourse communities, genres and English as an international language ». World Englishes 7, 211-220.

Swales, J. 1990. Genre Analysis : English in Academic \& Research Settings. Cambridge : Cambridge University Press.

Swales, John. 1996. « Teaching the conference abstract ». In Academic Writing Today and Tomorrow, Chapter 3. Yliopistopaino : Helsinki University Press, 45-59.

Swales, John. 1997. « The world's earliest-known technical texts : A brief note ». English for Specific Purposes 16, 151-152.

Toulmin, S.E. 1958. The Uses of Argument. Cambridge : Cambridge University Press.

Wittgenstein, L. 1961. Tractacus logico-philosophicus, Trad. P. Klossowski. Paris : Gallimard.

Sources documentaires techniques

Austin, G. 1984. Shreve's Chemical Process Industries. New-York : McGraw-Hill International Editions.

Cohendet, P., M.-J. Ledoux \& E. Zuscovitch. 1987. Les matériaux nouveaux : dynamique économique et stratégie européenne. Paris : Economica.

Perrin, R. \& J.-P. Scharff. 1993. Chimie industrielle. Paris : Masson.

\section{Sources électroniques}

Full text of all U.S. patents : QPAT-US <http://qpat2.qpat.com/info/help/about.html> (20 décembre 1997)

U.S. Patent and Trademark Office <http://www.uspto.gov> (15 janvier 1996)

Patent Application Data Entry Format <http://www.uspto.gov/web/offices/pac/dapp/sir/doc/ patappde.html> (6 février 1997)

Lexicon of Semiconductor Terms

<http://rel.semi.harris.com/docs/lexicon/preface.html> (20 février 1998)

Fuzzy Logic : a powerful new technology <http://www.fill.uni-linz.ac.at/fuzzy/introduction.html $>$ (15 mars 1998)

Fuzzy Logic and Fuzzy Expert Systems <http://www.cs.cmu.edu/Groups/AI/html/faqs/ai/fuzzy/ part1/faq-doc-22.html>(15 mars1998)

Fuzzy Systems : a tutorial <http://www.quadralay.com/Fuzzy/tutorial.html> (15 mars1998)

Vladimir Dimitrov \& David Russell : « The Fuzziness of Communication: A catalyst for seeking consensus ». <http://bart.northnet.com.au/ pfell/fuzcom.html> (consulté le 12 september 1998).

\section{ANNEXES}

\section{Corpus}

Brevet $\mathbf{n}^{\circ} 1: \mathrm{n}^{\circ} 947751$ Apparatus for the electrolytical decomposition of alkali-chlorid solutions

Original application filed May 4, 1906

Patented Jan. 25, 1910

Enjeu : mise au point d'un procédé de décomposition par électrolyse de produits dérivés 
du chlore, concerne des produits aussi divers que les papiers, les plastiques, les teintures et les pesticides.

$\rightarrow$ le début du siècle ouvre un champ d'action varié aux inventeurs.

Brevet $\mathbf{n}^{\circ} 2: \mathrm{n}^{\circ} 2,199,797$ Purification of chlorine

Original application filed September 13, 1937

Patented May 7, 1940 The Dow Chemical Company, Michigan

Enjeu :

$\rightarrow$ l'intérêt stratégique des techniques développées autour du chlore se confirment.

The Dow Chemical Company : Lorsque Herbert Dow mourut en 1931, son fils Willard devient président de Dow Chemical. Les temps s'annonçaient difficiles. Mais la société avait à sa tête une équipe de direction compétente techniquement. Le jeune président, ingénieur diplômé de l'Université de Michigan, avait déjà fait ses preuves, notamment en usine. L'association à parts égales des deux partenaires donne en 1931 Dow-Ethyl, productrice à la fois de chlore et de phénol : la société a lancé un programme de recherche sur les chlorophénols qui aboutissent à la mise au point des Dowicides, dérivés à fort pouvoir fongicide et germicide.

Le chlore

Aux besoins du chlore déjà reconnus pour la fabrication du tétrachlorure de carbone (CCl4) puis du trichloéthyléne, s'ajoutèrent ceux destinés à la récupération du brome de l'eau de mer. Le chlorhydrine d'éthylène est le précurseur de l'éthylène glycol ainsi que du caoutchouc Thiokol.

Le chlore entrait également dans la production du Néoprène, du chlorure de vinyle monomère et du caoutchouc chloré, produits encore nouveaux mais en plain développement.

Brevet $\mathbf{n}^{\circ} 3$ : $\mathrm{n}^{\circ} 3,073,005$ Composite fibers

Original application filed December 30, 1957

Patented January 15, 1963

Owens-Corning Fiberglas Corporation, a corporation of Delaware

Enjeu:

$\rightarrow$ mise au point d'une méthode destinée à améliorer les performances des fibres de verre et fibres de résines synthétiques, afin de les rendre plus extensibles et de les formater sous forme de spirales. (torsion)

Intérêt stratégique

$\rightarrow$ Au centre du concept de "grappe d'innovation », les matériaux composites occupent une place privilégiée.

1945-1960 : percées spectaculaires de la chimie d'après -guerre.

La période allant de 1945 à 1960 fut particulièrement féconde pour la chimie et, tout naturellement, pour les États-Unis, qui avaient, à l'abri des combats, développé considérablement leur potentiel industriel. L'Amérique était déjà en train de poser les jalons d'une chimie tirée du pétrole.

De plus, jusqu'en 1954, ils seront seuls à avoir, grâce au polyethylène et à l'oxyde d'ethylène, des débouchés importants dans leur éthylène pétrochimique, d'où leur suprématie dans le domaine des élastomères de synthèse.

Brevet $n^{\circ} 4: n^{\circ} 5,330,548$ Method for producing glass preform for optical fiber

Original application filed July 17, 1992

Patented January 19, 1994 
Sumitomo Electric Industries, Ltd., Osaka, Japan

Enjeu :

$\rightarrow$ mise au point d'une méthode de fabrication d'une préforme en verre transparent destinée à la fabrication des fibres optiques. L'amélioration par rapport aux procédés existants consiste à retirer le gaz de la préforme carbonée de manière à optimiser l'étape de vitrification.

Intérêt stratégique

-> Le premier matériau auquel les hommes ont naturellement pensé pour la transmission de la lumière fut bien sûr le verre. Dès le départ, les connaissances verrières traditionnelles influencèrent donc la recherche dans le domaine des fibres optiques. Le paramètre critique était l'absorption de la lumière dans le verre : une transparence suffisante ne fut finalement possible que grâce à l'utilisation de la silice. Toutefois, les procédés de production de fibres de verre employés jusqu'au début des années 90 ne permettaient pas l'utilisation de ce matériau Pour fabriquer de telles fibres, d'autres méthodes de production s'avéraient donc nécessaires, d'où le dépôt de brevets tels que celui-ci.

\section{Extraits du corpus}

Brevet $\mathbf{n}^{\circ} 1$ : 947,741 Electrolytical decomposition of alkali-chlorid solutions

Patented Jan. 25, 1910

The object of this invention is to provide an apparatus in which both surfaces of the mercury film are utilized for amalgamating surfaces, and instead of a horizontal film the mercury is caused to flow over the opposite surfaces of an upright conduit-plate at both sides of which the amalgamation takes place, there being anodes at opposite sides of said conduit-plate and diaphragms by which the bubbles of chlorin gas generated at the anodes are prevented from reaching the cathode formed by said conduit-plate and the steam of mercury flowing over the same.

Brevet $\mathbf{n}^{\circ} 2: 2,199,797$ Purification of Chlorine

Patented May 7, 1940

I therefore particularly point out and distinctly claim as my invention: The process of removing the organic impurities from impure chlorine gas containing less than about 1 per cent by weight of such impurities, which comprises introducing the impure chlorine gas into the base of a scrubbing tower below the level of the impure chlorine gas therein collected in a later step, permitting the chlorine gas to bubble through the said impure liquid chlorine and ascend in the tower against a downward flow of a relatively small amount of pure liquid chlorine produced in a later step and introduced into the upper portion of the said scrubbing tower whereby the organic impurities are absorbed by the liquid chlorine forming an impure liquid chlorine which collects in the base of the said scrubbing tower for use in the first step, withdrawing the so-treated chlorine gas from the upper portion of the scrubbing tower and impure liquid chlorine from the base thereof, liquefying the chlorine gas so withdrawn, and supplying the upper portion of the scrubbing tower with liquid chlorine from that so liquefied.

Brevet $\mathbf{n}^{\circ} 3$ : 3,073,005 Composite Fibers

Patented Jan. 15, 1963

[...] A feature of the invention lies in the fact that the fibers produced incorporate the desirable properties of straight fibers of the material from which they are made but additionally have an inherent curliness. 
A further feature of the invention lies in the adaptability of the invention to production of extremely fine curly fibers with conventional fiber attenuating means already well established in the art.

A still further feature of the invention lies in the lack of need for special treatment of the fibers subsequent to formation to impart the desired curliness thereto.

Another and still further feature of the invention lies in the fact that the surface forces of molten material utilized to produce conventional fibers lend themselves to establishment of an integral relationship between the compatible materials used to make up the curled fibers.

These and other objects and features of the present invention will become more apparent from the following description taken in conjunction with the accompanying drawings in which : [...]

Brevet $\mathbf{n}^{\circ} 4: 5,330,548$ Method for producing glass preform for optical fiber

Patented Jul. 19, 1994

Recently, it is ( ?) proposed to vitrify the glass soot preform under reduced pressure. By this method, the gas in the soot preform is removed by reduced pressure and it is ( ?) expected * that the gas does not remain in the vitrified preform.

In general, an apparatus for heating the soot preform under reduced pressure has a structure as shown in Fig.1. That is, the heating apparatus comprises a uniform heating furnace having a muffle tube 12 which surrounds a glass soot preform 11 and a heater 12 which surrounds the muffle tube, a heat shield 14 and a vacuum container 15 . The vacuum container is evacuated to the reduced pressure with a vacuum pump 17 through an outlet pipe 16 . After evacuation, the heater temperature is raised to vitrify the soot preform 11 in the muffle tube.

Using the above heating apparatus, a soot preform was vitrified by raising temperature under the temperature condition shown in Fig. 2 up to a vitrification temperature of, for example, $1550^{\circ}$ to $1650^{\circ} \mathrm{C}$. But, in some cases, bubbles remained in the vitrified preforms unexpectedly.*

* signal d'imprévu

\section{RÉSUMÉS}

Cet article aborde le problème des zones de flou dans les textes de brevets déposés aux ÉtatsUnis. Les outils linguistiques utilisés par les rédacteurs de textes de brevets convergent afin de mettre en oeuvre une stratégie de hedging. Le linguiste est amené à se demander si les hedges sont des mots et expressions destinés à créer du flou.

We investigate the problem of fuzziness in US patents. From examples of technical writing in early patent texts we see that technical language has complexified and strengthened and it gives rise to a strategy of hedging. From this we make a certain number of observations and wonder if hedges are "words whose job is to make things fuzzier". 
INDEX

Mots-clés : argumentation, brevet, enjeu économique, rhétorique (du discours technique), sémiosis hermétique, zone de flou

Keywords : economic pressure, fuzziness, patent, rhetoric (of technical discourse), rhetorical diffuseness

\section{AUTEUR}

\section{MONIQUE FROBERT-ADAMO}

Monique Frobert-Adamo enseigne l'anglais à l'IUT A Université Claude Bernard Lyon 1, où elle est responsable de la section langues et des Relations internationales, organisant des stages au Japon, aux États-Unis et en en Grande Bretagne. Ses axes de recherches sont l'analyse de la rhétorique du discours spécialisé dans une perspective systémique et les NTIC. Ses publications récentes comprennent des articles : «Hypermedia, Cybermedia », «Humour in Conference Language » et, en mars 1999, aux Éditions Ellipses « Thésaurus bilingue anglais-français des techniques de la chimie et du génie chimique ».Monique.Frobert@univ-lyon1.fr 